\title{
Follow-up of Newborns, Infants, and Children with Sepsis
}

\author{
P. Dahlem ${ }^{1} \quad$ P. Biggar ${ }^{2}$ \\ ${ }^{1}$ Department of Pediatrics, Medical Centre Coburg, Academic \\ Hospital of the University of Split, Coburg, Germany \\ 2 Department of Nephrology, Medical Centre Coburg, Academic \\ Hospital of the University of Split, Coburg, Germany \\ J Child Sci 2017;7:e38-e41.
}

\begin{abstract}
Address for correspondence P. Dahlem, MD, PhD, Department of Pediatrics, Medical Centre Coburg, Academic Hospital of the University of Split, Ketschendorferstr. 33, 96450 Coburg, Germany (e-mail: Peter.Dahlem@klinikum-coburg.de).
\end{abstract}

\begin{abstract}
Mortality in newborn infants and children with sepsis is high with survival rates of generally more than $50 \%$ in recent studies. Longitudinal follow-up studies have the potential to reveal short-term and lifelong physical, mental, and psychological sequelae. Although no comprehensive follow-up research has yet been performed, a small number of follow-up studies have shown that there is a considerable impact on the patients' lives and their families after hospital discharge. Health-related quality of

Keywords

- sepsis

- septic shock

- follow-up

- newborn infants

- children

- review life also seems to be affected; however, it does not correlate with severity of sepsis or handicap per se. Prematurely born infants, who can develop sequelae directly attributable to prematurity and its consequences, suffer differently from sepsis-related lifelong sequelae compared with older children. Fortunately, time may heal some wounds due to the effect of growth in children. In future, large centers should establish structural follow-up programs for clinical and research purposes to learn more about the needs of affected children and their families.
\end{abstract}

\section{Introduction}

Outcome and impact of pediatric sepsis can be measured as the number of complications during acute illness or number and characteristics of sequelae detected months or years after discharge. The so-called primary outcome parameter comprises frequent, clearly identifiable events in pediatric sepsis that vary greatly. Mortality may be as high as $57 \%, 29 \%$ having circulatory shock and $7 \%$ of children having organ failure. ${ }^{1}$ However, persisting physical and psychological abnormalities are also of interest and are important to the child and his/her family. Follow-up research should be addressed to find out whether or not these abnormalities change over time in the growing children. A significant natural difference between pediatric and adult sepsis is that sepsis afflicts the child at a particular stage of human development, whereas adults are fully grown and developed. Furthermore, it makes a difference

received

February 16, 2017 accepted after revision March 22, 2017
Issue Theme Pediatric Sepsis Biomarkers; Guest Editors: Peter Dahlem, MD, PhD,

Marijan Saraga, MD, PhD,

Walter L. Strohmaier, MD, PhD

DOI https://doi.org/ $10.1055 / \mathrm{s}-0037-1603893$ ISSN 2474-5871. whether sepsis occurs in an extremely premature baby with a basic risk for significant, life-long morbidity due to prematurity or older children in their age-dependent different stages of psychosocial development.

In addition, research should also encompass the parents, siblings, or even the whole family, as all have to handle the experience of having family members at the border of infection to the verge of death.

To address the impact of sepsis on physical and mental health after discharge, we searched the "PubMed" database for follow-up studies on pediatric and neonatal sepsis from the year 1990 until January 2017.

\section{Neonatal Sepsis}

We found only four studies in preterm infants and no appropriate follow-up study in term infants with bacterial

Copyright $\odot 2017$ Georg Thieme Verlag KG Stuttgart · New York 
sepsis. In preterm infants with neonatal sepsis, mortality may reach $30 \% .{ }^{2,3}$ However, performance of survivors in life is rarely studied. Specifically, in preterm infants, it is unclear whether bacterial sepsis contributes independently of the immediate consequences of prematurity to a worse longterm outcome.

In the United Kingdom, a thorough database analysis was performed of extremely low-birth-weight infants with a birth weight $<1,500 \mathrm{~g}$ born in the 1980s. This retrospective, uncontrolled observational study focused on neurological sequelae at the age of 18 months as a consequence of earlyonset neonatal sepsis. Twelve of the twenty children (60\%) with diplegia had suffered from neonatal sepsis. ${ }^{4}$ Furthermore, the relative risk of cerebral palsy increased fourfold compared with premature born infants having survived proven sepsis.

A nationwide prospective multicenter cohort study in Switzerland examined 541 very-low-birth weight infants born between 24 and 27 weeks of gestation over a study period of 7 years. ${ }^{5}$ At 2 years of age, neurodevelopmental outcome was assessed with Bayley Scales of Infant II. $^{6}$ Cerebral palsy was detected in $10 \%$ of those with cultureproven sepsis compared with $4 \%$ of children without infection. Specifically, impairment in neurodevelopment was found in 34 versus 23\%, respectively. Proven sepsis was identified as an independent risk factor compared with a priori sequelae of prematurity for both end points, i.e., cerebral palsy and neurodevelopmental impairment.

A recently presented retrospective study of 437 very-lowbirth weight infants in Germany covered a follow-up period of 10 years. ${ }^{7}$ In contrast to the previously mentioned study, the frequency of cognitive impairment and cerebral palsy in this vulnerable age group was not dependent on the occurrence of sepsis but on neurological damage, such as intraventricular hemorrhage III, periventricular hemorrhage, periventricular leucomalacia, and furthermore on dependence on the length of mechanical ventilation.

Not only early-onset but also late-onset neonatal sepsis might cause health-related impairment later in life. Investigators of a Dutch cohort examined the outcome of verylow-birthweight infants at school age. ${ }^{8}$ Fifty-one infants with a culture-proven sepsis were included. Follow-up assessment included standardized motor and cognitive tests. Follow-up was performed at school age, which is a very important first landmark in human development. Thirty-two infants were eligible for analysis. The main finding was that the study population showed on average significant motor function impairment and lower intelligence quotient (IQ). Significant deficits in attention and verbal memory were detected compared with controlled preterm infants without sepsis. Late-onset sepsis with gram-negative bacteria correlated with the worst outcome. This is the only study that analyzed the outcome related to different bacterial strains.

Up to now, no studies have focused on sequelae in term infants with bacterial sepsis. Specifically, successfully treated, previously healthy infants should be followed to determine lifelong physical and mental compromise.

\section{Pediatric Sepsis}

Pediatric bacterial sepsis afflicts children outside the newborn period until the age of 18 years. Almost all children with bacterial sepsis are treated in a pediatric intensive care unit (PICU) and most of them are discharged to home. In general, the impact of PICU admission and critical illness on the survivors and their parents is studied by appropriate follow-up research not only on physical and psychological abnormalities but also on the quality of life standards. Although, the majority of children survive without sequelae, a considerable number of survivors show life-long physical and psychological sequelae. ${ }^{9}$

Utilizing structured medical examinations, Knoester et al examined 186 previously healthy children unexpectedly admitted to the PICU. Three months after discharge, survivors often suffered from multiple physical and neurocognitive problems with an overall morbidity of $38 \%$. Most frequent problems were respiratory (12\%), neurological (26\%), and miscellaneous (8\%). ${ }^{10}$ Also, health problems with a numerically lower frequency but with a high impact on quality of life were found, such as skin scars (9\%), hoarseness (3\%), postthrombotic syndrome (4\%), and tracheotomy (2\%).

Knowledge of the impact of sepsis on the quality of life is imperative. To answer this question, 81 children, in different age groups, discharged from a Dutch tertiary PICU were evaluated regarding standardized health-related quality of life (HRQoL) questionnaires. ${ }^{11}$ Within the first 12 months after discharge, the children showed highly significant lower values for HRQoL compared with age-matched healthy controls. The lowest scores were achieved for the domains of liveliness and problem behavior. Interestingly, health problems evaluated at 3 and 9 months after discharge showed a trend to improve. Specifically, physical abnormalities improved due to the capacity of the body to still grow. ${ }^{11}$

Of course, the first few months after a life-threatening illness constitute a very intense period for the child and its family. Not only the child, but also the parents may apparently have to cope with the consequences. ${ }^{12}$ However, many traumatic moments remain vividly entangled in mixed emotions, such as self-recrimination and fear of losing the loved child on one hand, and hopes, beliefs, expectations, and happiness that the child has survived, on the other hand.

Bacterial sepsis, as one of the most frequent PICU diagnoses and issue of this review, has not been thoroughly studied despite being an independent risk factor determining the outcome. Available data are scarce and consist mostly of follow-up of children with meningococcal septic shock (MSS) due to its endemic occurrence in many countries. ${ }^{13,14}$

At a follow-up after 2 years from discharge, 55\% of 47 children (28 boys and 19 girls) with MSS admitted to a Dutch tertiary PICU suffered from skin scars and $4 \%$ had to cope with amputations. $^{15}$ Additionally, $45 \%$ confirmed chronic complaints, such as pain, behavioral problems, and motor skill problems. The severity of MSS and the number of complaints correlated with lower scores in the HQRoL questionnaires. Parents were also affected. Not surprisingly, $15 \%$ required professional psychiatric therapy due to anxiety and depression. 
Time may not heal all wounds. Ten years after acute illness, important sequelae have been found. In a Dutch follow-up clinic for children with MSS, 120 children with a follow-up age of 14.5 years were investigated with standardized physical and mental examinations. ${ }^{12,16}$ Psychological examination included emotional, behavioral, and intelligence tests. Physical examination focused on the specific problems of meningococcal infection, such as skin scaring, orthopaedic problems, and neurological testing. Finally, the HRQoL questionnaires revealed one or more sequelae at follow-up examination in $61 \%$ of children. Neurological impairment occurred in $35 \%$ of children, which included headache, epilepsy, and focal deficits. Health status was reduced in almost all participants. Interestingly, the investigators also focused on correlations between different outcome parameters. Surprisingly, they found no correlation between major physical abnormalities such as skin scaring or limb amputation and neurological, cognitive, or behavior impairment. This is remarkable and might be related to variable phenomenon of resilience of the children and their whole families.

Children are dependent and embedded into their families. However, it is not clear how young adults still suffer from complaints related to a period of severe sepsis during childhood. Vermunt et al assessed psychological outcome at a mean age of 23 years and at a mean follow-up interval of 13 years. ${ }^{17}$ Up to $20 \%$ of 58 eligible patients still struggled with behavioral, emotional, and social problems and scored lower during intelligence tests.

Some investigators questioned whether organ dysfunction, such as renal and cardiac failure, which is frequently present during MSS, recovers or persists. Twelve of the 21 children with acute renal failure treated with dialysis during MSS showed renal dysfunction, such as reduced glomerular filtration rate, proteinuria, or hypertension 4 years after discharge. ${ }^{18}$ However, these abnormalities required no therapy.

In pediatric septic shock, circulatory failure is a very common life-threatening phenomenon, and intravenous catecholamines are used in a majority of patients. ${ }^{13,19}$ Myocardial dysfunction may be present in approximately $50 \%$ of patients, and therefore, a complete restoration of cardiac function is of great interest. ${ }^{19}$ Fortunately, as shown by Knoester et al in 108 children, only a small number of children showed minor cardiac abnormalities after more than 6 months of discharge. ${ }^{20}$ All had a normal medical history and physical examination, and only 6\% showed abnormalities either at electrocardiography or echocardiography. None of them needed therapy.

Due to the strong interaction between inflammation, coagulation, and compromise of peripheral perfusion, children with MSS have a high risk for orthopaedic problems such as amputation and growth plate arrest. ${ }^{21}$ At follow-up, attention should be given to differences in the growth of limbs. If differences persist, lengthening osteotomy or ankle corrections may be necessary with special care after amputation.

School-aged children might be of particular interest for follow-up research. The research would help to understand the reason for the deterioration of their school performance after critical illness. In a study of 88 children, Als et al performed neuropsychological assessment within the first 12 months after sepsis. ${ }^{22}$ Additionally, academic performance was tested comparing the educational needs and cognitive function of the children assessed routinely before and on follow-up after illness. Children with sepsis scored worse in neuropsychological function tests compared with the healthy controls. Interestingly, they had the second lowest values compared with other subgroups of children admitted to PICU. Only children with meningoencephalitis performed worse. After returning to school, teachers found deficits in academic skills in approximately $20 \%$ of children.

The main topic of this special edition is biomarkers of pediatric sepsis.Theoretically, outcome might correlate with the levels of specific biomarkers of inflammation.

Recently, new biomarkers have been investigated in a single center cohort, whether or not they correlate with short-term outcomes. A total of 90 patients $<18$-years-old were included in this study; 30 with severe sepsis or septic shock were compared with 30 age-matched febrile and 30 age-matched healthy controls. The ratio of matrix metalloproteinase 9 to tissue inhibitor of metalloproteinase showed mid-regional pro-atrial natriuretic peptide, and adipocyte fatty acid-binding proteins were not only indicative of the presence of sepsis, but also showed correlation with poorer outcome and length of hospital stay. ${ }^{23}$ However, to date, no comprehensive data on the prognostic power of biomarkers exist.

\section{Conclusion}

The impact of bacterial sepsis on the child and its family is tremendous and might differ between the various pediatric age groups ranging from preterm and term neonates to school-aged children, adolescents, and young adults. Follow-up on health-related quality of life after critical illness and the correlation between the severity of critical illness and physical/psychological handicaps are very important areas for research. ${ }^{1}$ Not only the child but the parents and the whole family are affected by the acute event and should be examined on follow-up. There are no uniformly applicable HRQoL tests, and test intervals are not standardized.

A subgroup analysis regarding the bacterium found on culture might also be of interest. Each bacterium interacts with the immune system in a different way. ${ }^{24}$ Theoretically, it is conceivable that different bacterial strains may influence longterm outcomes in different ways. However, the incidence of infections caused by different strains is generally so low that a study appears impracticable. Regarding MSS, which still occurs in endemic areas, fortunately, the number of children with MSS is declining due to nationwide vaccination programs. ${ }^{13,25}$

Research on future outcome should also investigate the correlation of biomarkers, such as acute phase proteins and others, with physical and mental sequelae.

\section{References}

1 Menon K, McNally JD, Zimmerman JJ, et al. Primary outcome measures in pediatric septic shock trials: a systematic review. Pediatr Crit Care Med 2017;18(03):e146-e154 
2 Stoll BJ, Hansen N, Fanaroff AA, et al. Late-onset sepsis in very low birth weight neonates: the experience of the NICHD Neonatal Research Network. Pediatrics 2002;110(2, Pt 1):285-291

3 Stoll BJ, Hansen N, Fanaroff AA, et al. Changes in pathogens causing early-onset sepsis in very-low-birth-weight infants. N Engl J Med 2002;347(04):240-247

4 Wheater M, Rennie JM. Perinatal infection is an important risk factor for cerebral palsy in very-low-birthweight infants. Dev Med Child Neurol 2000;42(06):364-367

5 Schlapbach LJ, Aebischer M, Adams M, et al; Swiss Neonatal Network and Follow-Up Group. Impact of sepsis on neurodevelopmental outcome in a Swiss National Cohort of extremely premature infants. Pediatrics 2011;128(02):e348-e357

6 Bayley N. Manual for the Bayley Scales of Infant Development, 2nd ed. San Antonio, TX: The Psychological Corporation; 1993

7 Voss W, Hobbiebrunken E, Ungermann U, Wagner M, Damm G. The development of extremely premature infants. Dtsch Arztebl Int 2016;113(51-52):871-878

8 van der Ree M, Tanis JC, Van Braeckel KN, Bos AF, Roze E. Functional impairments at school age of preterm born children with late-onset sepsis. Early Hum Dev 2011;87(12):821-826

9 Knoester H, Grootenhuis MA, Bos AP. Outcome of paediatric intensive care survivors. Eur J Pediatr 2007;166(11):1119-1128

10 Knoester H, Bronner MB, Bos AP. Surviving pediatric intensive care: physical outcome after 3 months. Intensive Care Med 2008; 34(06):1076-1082

11 Knoester H, Bronner MB, Bos AP, Grootenhuis MA. Quality of life in children three and nine months after discharge from a paediatric intensive care unit: a prospective cohort study. Health Qual Life Outcomes 2008;6:21. Doi: 10.1186/1477-7525-6-21

12 Buysse CM, Vermunt LC, Raat H, et al. Surviving meningococcal septic shock in childhood: long-term overall outcome and the effect on health-related quality of life. Crit Care 2010;14(03): R124. Doi: $10.1186 /$ cc9087

13 Theilen U, Wilson L, Wilson G, Beattie JO, Qureshi S, Simpson D; Guideline Development Group. Management of invasive meningococcal disease in children and young people: summary of SIGN guidelines. BMJ 2008;336(7657):1367-1370

14 Hart CA, Thomson AP. Meningococcal disease and its management in children. BMJ 2006;333(7570):685-690

15 Buysse CM, Raat H, Hazelzet JA, Hop WC, Maliepaard M, Joosten KF. Surviving meningococcal septic shock: health consequences and quality of life in children and their parents up to 2 years after pediatric intensive care unit discharge. Crit Care Med 2008; 36(02):596-602

16 Buysse $\mathrm{CM}$, Raat $\mathrm{H}$, Hazelzet JA, et al. Long-term health status in childhood survivors of meningococcal septic shock. Arch Pediatr Adolesc Med 2008;162(11):1036-1041

17 Vermunt LC, Buysse CM, Joosten KF, et al. Survivors of septic shock caused by Neisseria meningitidis in childhood: psychosocial outcomes in young adulthood. Pediatr Crit Care Med 2011; 12(06):e302-e309

18 Slack R, Hawkins KC, Gilhooley L, Addison GM, Lewis MA, Webb NJ. Long-term outcome of meningococcal sepsis-associated acute renal failure. Pediatr Crit Care Med 2005;6(04): 477-479

19 Rudiger A, Singer M. Mechanisms of sepsis-induced cardiac dysfunction. Crit Care Med 2007;35(06):1599-1608

20 Knoester H, Sol JJ, Ramsodit P, Kuipers IM, Clur SA, Bos AP. Cardiac function in pediatric septic shock survivors. Arch Pediatr Adolesc Med 2008;162(12):1164-1168

21 Park DH, Bradish CF. The management of the orthopaedic sequelae of meningococcal septicaemia: patients treated to skeletal maturity. J Bone Joint Surg Br 2011;93(07):984-989

22 Als LC, Nadel S, Cooper M, Pierce CM, Sahakian BJ, Garralda ME. Neuropsychologic function three to six months following admission to the PICU with meningoencephalitis, sepsis, and other disorders: a prospective study of school-aged children. Crit Care Med 2013;41(04):1094-1103

23 Alqahtani MF, Smith CM, Weiss SL, Dawson S, Ralay Ranaivo H, Wainwright MS. Evaluation of new diagnostic biomarkers in pediatric sepsis: matrix metalloproteinase-9, tissue inhibitor of metalloproteinase-1, mid-regional pro-atrial natriuretic peptide, and adipocyte fatty-acid binding protein. PLoS One 2016;11(04): e0153645. Doi: 10.1371/journal.pone.0153645

24 Gasparini R, Mennini FS, Panatto D, et al. How can the results of Health Technology Assessment (HTA) evaluations applied to vaccinations be communicated to decision-makers and stakeholders? The ISPOR Rome Chapter Project. J Prev Med Hyg 2015; 56(04):E150-E154

25 Paireau JC, Broutin H, Grenfell B, Basta NE. Global trends in seasonal dynamics of bacterial meningitis: a time-series analysis. 20th International Pathogenic Neisseria Conference, September 4-9. Manchester, United Kingdom; 2016:109 